\title{
BMJ Open Protocol for a mixed methods study of hospital readmissions: sensemaking in Veterans Health Administration healthcare system in the USA
}

\author{
Lauren S Penney, ${ }^{1,2}$ Luci K Leykum, ${ }^{1,2,3}$ Polly Noël, ${ }^{4}$ Erin P Finley, ${ }^{1,2,5}$ \\ Holly Jordan Lanham, ${ }^{1,3,4}$ Jacqueline Pugh ${ }^{1,2}$
}

To cite: Penney LS, Leykum LK, Noël P, et al. Protocol for a mixed methods study of hospital readmissions: sensemaking in Veterans Health Administration healthcare system in the USA. BMJ Open 2018;8:e020169. doi:10.1136/ bmjopen-2017-020169

- Prepublication history and additional material for this paper are available online. To view these files, please visit the journal online (http://dx.doi. org/10.1136/bmjopen-2017020169).

Received 18 0ctober 2017 Revised 9 February 2018 Accepted 20 February 2018

Check for updates

${ }^{1}$ Research Service, South Texas Veterans Health Care System, San Antonio, Texas, USA ${ }^{2}$ Department of Medicine, University of Texas Health Science Center San Antonio, San Antonio, Texas, USA

${ }^{3}$ Department of Information, Risk and Operations Management,

McCombs School of Business, University of Texas, Austin,

Texas, USA

${ }^{4}$ Department of Family and Community Medicine, University of Texas Health Science Center San Antonio, San Antonio, Texas, USA

${ }^{5}$ Department of Psychiatry, University of Texas Health Science Center San Antonio, San Antonio, Texas, USA

Correspondence to

Dr Lauren S Penney;

Lauren.Penney@va.gov

\section{ABSTRACT}

Introduction Effective delivery of healthcare in complex systems requires managing interdependencies between professions and organisational units. Reducing 30-day hospital readmissions may be one of the most complex tasks that a healthcare system can undertake. We propose that these less than optimal outcomes are related to difficulties managing the complex interdependencies among organisational units and to a lack of effective sensemaking among individuals and organisational units regarding how best to coordinate patient needs.

Methods and analysis This is a mixed method, multistepped study. We will conduct in-depth qualitative organisational case studies in 10 Veterans Health Administration facilities ( 6 with improving and 4 with worsening readmission rates), focusing on relationships, sensemaking and improvisation around care transition processes intended to reduce early readmissions. Data will be gathered through multiple methods (eg, chart reviews, surveys, interviews, observations) and analysed using analytic memos, qualitative coding and statistical analyses. We will construct an agent-based model based on those results to explore the influence of sensemaking and specific care transition processes on early readmissions. Ethics and dissemination Ethical approval has been obtained through the Institutional Review Board of the University of Texas Health Science Center at San Antonio (approval number: 14-258 hour). We will disseminate our findings in manuscripts in peer-reviewed journals, professional conferences and through short reports back to participating entities and stakeholders.

\section{INTRODUCTION}

Complex systems cannot be understood by breaking their processes down into component parts or into individuals' jobs, even though this is often our first response to solving complicated problems in healthcare. ${ }^{12}$ Effective healthcare delivery requires effective management of interdependencies between socially distinct professions and between organisational units with unique perceived purposes and purviews. Within well-integrated systems, patients navigating
Strengths and limitations of this study

- Using Eisenhardt's recommendations for building theory from case studies, this study samples 10 sites with a minimum of 2000 discharges per year, all of which have attempted efforts to improve hospital-to-home care transition processes and have either worsening or improving hospital readmission rates over a 5-year period, allowing us to explore organisational characteristics leading to these performance patterns.

- For each site, we create an in-depth qualitative organisational case study of relationships, sensemaking and improvisation around care transition processes, from which we will build an agent-based model to explore how system elements may impact hospital readmission rates and identify potential leverage points for new types of interventions.

- Limitations include the single point in time data collection, all facilities are drawn from a single healthcare system (the Veterans Health Administration) and the study is observational rather than interventional.

unit boundaries should feel like system components form a continuum that communicate and cooperate for the explicit purpose of patient wellness.

As the largest integrated healthcare system of the USA, the Veterans Health Administration (VHA) is theoretically positioned to deliver integrated care along such a continuum. Despite this, VHA's performance has been similar or worse than Medicare providers with regard to outcomes reflecting complex interdependencies, such as unplanned hospital readmissions. ${ }^{3}$ We propose that these less than optimal outcomes are related to difficulties managing the complex interdependencies among VHA organisational units and to a lack of effective sensemaking among individuals and organisational units regarding how best to coordinate Veteran needs. 


\section{Early readmissions as a persistent problem}

Hospital readmissions continue to receive significant attention as a source of potential waste and a marker of poor quality. Although the policy emphasis on readmissions is recent, ${ }^{4}$ early readmissions have been proposed as a quality indicator for at least 22 years. ${ }^{5}$ Numerous studies assessing the extent of preventability of early readmissions have had widely varying estimates: $5 \%-79 \% .^{6-8}$

Readmission rates have been declining but are still felt to be unacceptable. ${ }^{9}$ VHA hospital-wide risk-adjusted 30-day readmission rates gradually dropped 3\% from 1997 to $2010(16.5 \%-13.8 \%),{ }^{10}$ and have remained around 13\% (IPEC readmission cube on VHA Support Service Center, accessed 19 May 2017).

Why has reducing early hospital readmissions been such a persistent challenge? Reducing readmissions within 30 days may be one of the most complex tasks in a healthcare system. First, success depends on the intersection, coordination and collaboration of many parts of the system Second, patients and their caregivers are in control of many of the factors that will determine their ability to stay out of the hospital, and healthcare delivery systems may not recognise the challenges faced postdischarge. Third, with a focus on shortening hospital length of stay, assumptions have been made about who is responsible for different aspects of care, with gaps occurring when expectations are not congruent. Fourth, there is a dearth of geriatricians who might have more insight into frail patients' needs and be better equipped to deal with the large numbers of chronically ill elderly. ${ }^{11}$ Fifth, due to ongoing fragmentation of provider-patient relationships, there may be both a lack of recognition of and communication regarding the need for palliative care. Finally, technologies and processes that prolong life may require a greater number of appropriate hospital admissions over an individual's life course.

Given the complexity of understanding all elements contributing to readmissions, it is no surprise that preventing early readmissions remains a challenging healthcare issue.

\section{Risk prediction models for readmissions}

One approach to reduce readmission rates has been to implement risk prediction models to identify and target interventions towards those most at risk for early readmission. Kansagara et $a l^{12}$ reviewed 26 unique models. They concluded that most readmission risk prediction models performed poorly and as yet are not useful in clinical settings. This finding was corroborated by a systematic review by Zhou et al, ${ }^{13}$ which found that while risk prediction models are growing in number and condition specificity, they show only moderate discriminative ability. These models typically focused on risk characteristics of the patients and not characteristics of institutional behaviour that might put patients at risk.

\section{Care transitions studies}

Another approach to reducing readmission rates is through care transition interventions. Hansen et $a l^{14}$ found that of 16 randomised, controlled trials of interventions to improve 30-day rehospitalisation rates, only 5 documented statistically significant improvement in reducing rehospitalisations. Four of these five tested multicomponent discharge bundles, however 11 other randomised controlled trials, some of which also used bundles with similar elements, failed to show improvements. Leppin $e t a l^{15}$ found the majority of reviewed trials (38 of 42) did not have a significant effect on readmissions; however, studies with five or more unique activities in the intervention were more effective at reducing readmissions than those with two or more activities. One interpretation of these mixed findings from the perspective of complexity science is that interventions focus on breaking down processes into component parts or on changing the behaviours of individuals (assigning specific individuals to specific tasks) but do not address the interdependencies and boundary crossings that make the transitions so difficult.

Despite the ambiguity of the evidence and because of the burden of readmission for both the patient and the system, many individual VHA facilities are trying some of the more promising of the above models (eg, Project RED, Project BOOST). There have also been VHA-sponsored efforts, such as to address chronic heart failure readmissions ${ }^{16}$ and to enact transition management initiatives, and nationwide policies to conduct discharge medication reconciliation and to conduct postdischarge follow-up calls. However, there are few care transition elements mandated to be implemented across VHA facilities.

\section{Complexity science as a theoretical lens for understanding why reducing readmissions is so difficult}

The application of complexity science to healthcare systems can provide new insights to the issue of readmissions. Defining characteristics of complex adaptive systems are diverse learning agents who interact non-linearly and who self-organise. These complex systems co-evolve with their environment and have emergent properties that are not predictable. Due to the systems' non-linearity, inputs and outputs are not necessarily proportional. ${ }^{17}$ Even though organisations might implement care transition programmes, the amount of effort put into their programmes is not necessarily proportional to readmission rate outcomes.

The inherent non-linearity of complex systems also leads to uncertainty. This may be particularly true during transitional periods for patients, when patients' recovery is not yet assured, the home environment is often not well known to the staff and the possibility of developing a relapse is significant. In these situations, uncertainty is compounded. ${ }^{18}{ }^{19}$ Implementing new initiatives and changing processes also introduce uncertainty. An implication of this is that improvement efforts need to focus 


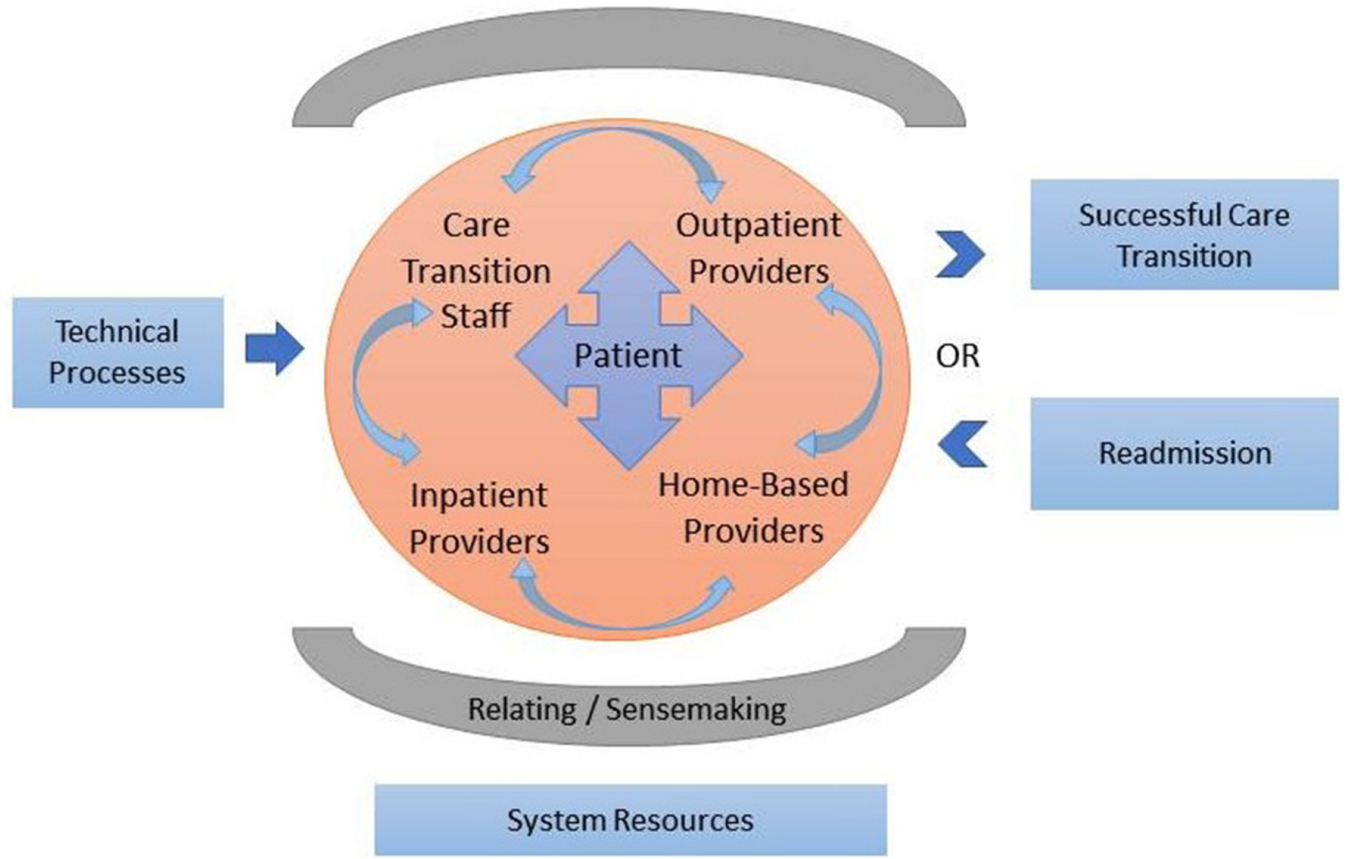

Figure 1 Model of care transitions.

on process of care, and on the relationships between and interdependencies among healthcare providers. ${ }^{1} 20$

\section{Relationships, sensemaking and improvising}

Relationships among healthcare workers are the foundation for the social activities that occur during patient care, like transitions of care. The framework of work relationships by Lanham et al proposes that seven characteristics define effective relationships in healthcare: trust, mindfulness, heedfulness, respectful interaction, diversity, social and task relatedness and rich and lean conversation. ${ }^{21}$ These characteristics interact with how individuals and groups of providers reflect, make sense and learn in ways that shape the quality of patient outcomes. Through relationship infrastructure, care transitions staff can coordinate as an effective, interdependent group in patient care. However, fostering relationships to improve care delivery is not something to which healthcare organisations have traditionally paid attention, even though data speak to its importance. ${ }^{21-23}$

Differences in relationship infrastructures across services, teams and organisations may help explain the varying impacts of care transition interventions. The relationship infrastructure can give way to activities, such as sensemaking and improvising, which help providers and other organisational staff manage uncertainties and stressors. In sensemaking, people assimilate information, reach conclusions and take steps to act. ${ }^{24}$ In the inpatient setting, sensemaking can occur in relation to individual patient diagnosis and care, as well as understanding more broadly patient illness trajectories and how their condition changes over time. ${ }^{25}$

Preventing early readmissions via sensemaking involves multiple sets of individuals interacting to make sense beyond the physician team. Our model below summarises these interdependencies (figure 1). The trajectory of the patient's illness needs to be understood as it continues in the home or next institutional environment and in relation to how well the home environment meets patient posthospitalisation needs, what actual supports need to brought together, the level of understanding of the patient and/or caregiver of the self-management that will need to occur, understanding of funding mechanisms and more. While checklists provide reminders of what needs to be done, they do not necessarily help providers make sense of what needs to be done for whom, or when or how to engage others to help.

Improvising is varying what one does based on the context and situation at hand. ${ }^{26}{ }^{27}$ Physicians describe the importance of improvisation amid new or uncertain situations in patient care. ${ }^{27}$ Thus, improving care transitions teams' ability to improvise may be a powerful strategy for targeting activities to the needs of individual patients and decreasing readmissions.

\section{Project aim}

We are studying care transition interventions aimed at reducing early readmissions as an exemplar of processes requiring a high level of interdependencies and sensemaking. By investigating VHA facility cases that have attempted interventions to improve care transitions and have had either improvement or worsening in their readmission rates, we will improve our understanding of the care transition processes themselves and the sensemaking within the organisation needed to implement change when there is no single part of the organisation responsible for the outcome.

- Objective 1: conduct in-depth qualitative, organisational case studies to explore relationships, sensemaking and improvisation in six facilities with 
improving and four facilities with worsening early readmissions rates between fiscal years 2006 and 2011, all of which engaged in care transition interventions to improve early readmissions.

- Objective 2: extend learning from and enhance generalisability of the case studies, using agent-based model (ABM) to simulate facilities implementing care transition innovations and to explore both specific care transition processes and elements of sensemaking as they prevent early readmissions, or not, as possible system outcomes.

\section{METHODS AND ANALYSIS \\ Study design overview}

We are conducting a mixed method, multistepped study using concurrent triangulation. It will be conducted in two parts: the first part will be an in-depth qualitative organisational case study; the second part will be constructing an $\mathrm{ABM}$ based on those results.

\section{Objective 1. Organisational case studies}

Case sample and individual recruitment within cases

Given that the intent of the study is to build or extend theory, not to test existing theory, we are using Eisenhardt's recommendations with regard to sampling for case studies in her methodological review, 'Building theories from case study research' ${ }^{28}$ In this context, cases are chosen on theoretical grounds and not for statistical reasons. Cases may be chosen to replicate previous cases or extend emergent theory or they may be chosen to fill theoretical categories and provide examples of polar types, in which the process of interest is "transparently observable'. ${ }^{28}{ }^{29}$ Random selection is neither necessary nor even preferable. The goal of the theoretical sampling is to choose cases which are likely to replicate or extend the emergent theory. In this spirit, our criteria for case selection concerned facility size, trending 5 -year readmission rates and documented care transition improvement efforts (table 1).

Within each facility case, individuals will be recruited to participate in interviews, focus groups, observations and/or surveys using purposive sampling. ${ }^{30}$ Purposive sampling allows us to identify and recruit individuals with specific experiences and knowledge that will inform our case building. We will use information from facility websites (eg, organisational charts, service rosters) and the VA's Microsoft Outlook contact list to identify individuals occupying specific roles. During site visits, snowball and convenience sampling will also be used to identify people with knowledge of site care transition innovations and experience with care transition practices.

Potential participants will be invited to participate through email and/or face-to-face. Specific forms of sampling and recruitment will vary based on data collection activity (table 2). Note, recruitment for one activity does not preclude recruitment for other activities. For example, a hospitalist might be engaged in an interview as well as an observation of her medicine rounds. At each site, investigators will aim to balance recruiting to obtain diverse, representative perspectives and to generate deeper knowledge about specific experiences.

All providers and staff recruited to participate in interviews, focus groups, observations and surveys will be

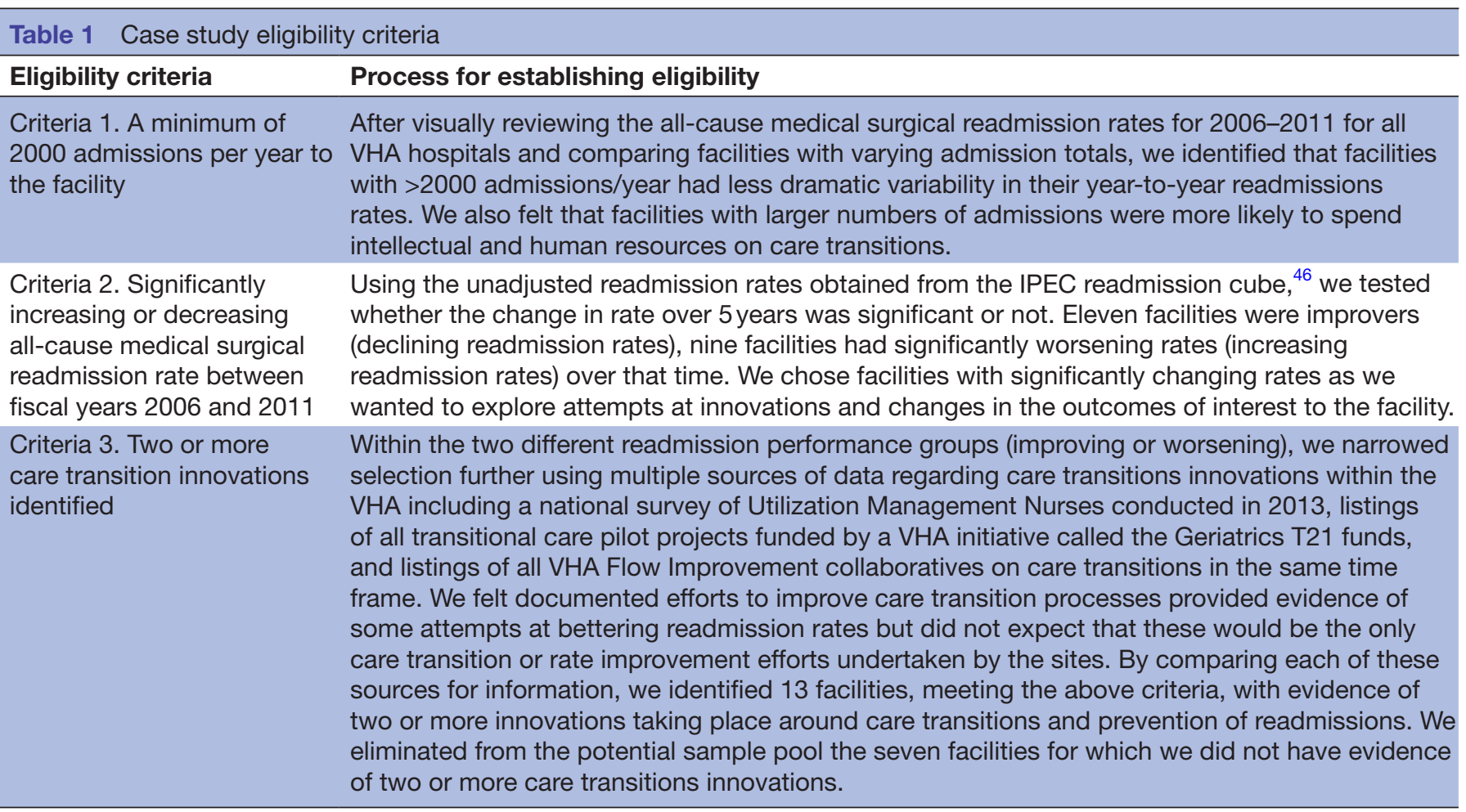


Table 2 Participant recruitment for each case study site

\begin{tabular}{|c|c|c|}
\hline Activity & Population & Description of recruitment \\
\hline Interviews & $\begin{array}{l}\text { Service leaders } \\
(n=\sim 10)\end{array}$ & $\begin{array}{l}\text { Individuals from medicine, nursing, social work, pharmacy and primary care leadership (ie, } \\
\text { service chiefs and supervisors) will be identified through organisational charts available on } \\
\text { facility websites or sharepoints, the VHA outlook contact list or by other staff at the facility. } \\
\text { They will be contacted by phone or by email to participate in interviews. }\end{array}$ \\
\hline Chart reviews & $\begin{array}{l}\text { Patients } \\
(n=10)\end{array}$ & $\begin{array}{l}\text { Project staff and investigators will review the charts of a random selection of } 10 \text { veterans } \\
\text { admitted to the facility's hospital within the } 3-6 \text { months before the scheduled site visit. Five of } \\
\text { the veterans will have had } 30 \text {-day readmissions following their index admissions and five of } \\
\text { them will have not. All } 10 \text { veterans must meet the following inclusion criteria at the time of the } \\
\text { index admission: (a) inpatient or outpatient contact in the previous year with a VHA provider; } \\
\text { (b) a Charlson Comorbidity Index }{ }^{47} \text { of two or more; (c) discharge from a general medicine } \\
\text { unit at the case study hospital within the sampling period; (d) discharge diagnosis of chronic } \\
\text { obstructive pulmonary disease, chronic heart failure and/or pneumonia and (e) discharge to } \\
\text { home. Patients are excluded if they are discharged to a long-term care or skilled facility. For } \\
\text { each site, a VA data analyst will provide the team with a sample of the first } 10 \text { readmitted } \\
\text { and } 10 \text { non-readmitted patients meeting these criteria. The project coordinator will verify } \\
\text { that these patients meet eligibility criteria and assign the first five in each group which meet } \\
\text { eligibility criteria to be reviewed. A waiver of consent was obtained for the sample of patients } \\
\text { for whom we conduct chart reviews. }\end{array}$ \\
\hline Interviews & $\begin{array}{l}\text { Frontline } \\
\text { providers } \\
(\mathrm{n}=15-20)\end{array}$ & $\begin{array}{l}\text { We will sample one to four providers from each of the following roles: hospitalists, inpatient } \\
\text { medicine nurses, inpatient social workers, pharmacists who deal with discharge education } \\
\text { and supply of medications to patients on discharge, primary care team providers and, when } \\
\text { present, dedicated care transition staff (eg, patient care coordinators). Depending on each } \\
\text { site's processes and programmes, interviews may also be held with representative staff from } \\
\text { palliative care, subspecialty care (eg, geriatrics, cardiology), telecare, utilisation management } \\
\text { and others as appropriate. }\end{array}$ \\
\hline
\end{tabular}

Focus groups Frontline providers $(n=1-2)$

$\begin{array}{cc}\text { Observations } & \text { Frontline } \\ & \text { providers } \\ (n=17-30)\end{array}$
providers $(n=15)$
Surveys Frontline

One to two focus groups, comprised 4-10 individuals, will be held at each site. For each focus group, the team will aim to recruit one to two staff to represent the following roles: hospitalists, nurses, social workers, pharmacists and any roles important to care transitions at that site (eg, patient care coordinators, utilisation management nurses). Investigators will recruit frontline staff using snowball and quota sampling methods.

Staff participating in discharge planning, performing care transition tasks (eg, discharge education) and doing day-to-day work on medicine units (eg, rounds) will be eligible for observation. Investigators will purposively recruit participants for observations before the site visit (eg, through email) and face-to-face during the site visit prior to the start of observations. The specific types of activities observed and number of times they are observed will vary depending on the facility, but the team will broadly aim to observe three to six medicine rounds, three to six discharge planning meetings, four med-surg unit observations, three to six job role shadowing and four to eight patient discharge educations. Observation lengths will also vary, from $10 \mathrm{~min}$ (eg, patient discharge education) to 3 hours (eg, medicine rounds). During observations, as necessary, researchers will identify themselves to obtain verbal consent from other patients, staff and other individuals that enter the field of observation once it has commenced. Investigators will use discretion to cease observations if they determine an individual may not be in a position to provide informed consent (eg, a critically ill patient). Data collection will cease if any person declines to be observed.

Members of the inpatient care transition teams (eg, hospitalists, social workers, nurses, pharmacists) and any frontline staff members with a direct role in care transitions (eg, primary care nurses and physicians) will be invited to participate in an anonymous survey. They will be identified during data collection activities (eg, observing discharge planning meetings, individual interviews), and invited to participate either by email or in person. Everyone encountered who is eligible to participate will be recruited. Surveys can be filled out online (through REDCap) or by handing in a paper copy, neither form collects identifying information and investigators will not make any notes about who turns in paper forms of the survey.

$\begin{array}{lll}\text { Interviews } & \begin{array}{l}\text { Patients } \\ (n=5)\end{array} & \begin{array}{l}\text { Five patients being discharged from medicine units to home will be recruited for interviews. } \\ \text { Patients will be sampled using convenience methods and identified by frontline staff. }\end{array} \\ \text { Exit debrief } & \begin{array}{l}\text { Facility leaders } \\ (n=2-8)\end{array} & \begin{array}{l}\text { During early email communications with site representatives, facility leadership will be asked } \\ \text { to attend an hour long exit debrief on the last day of the team's site visit. Facility directors and } \\ \text { chiefs of staff will be invited, along with anyone else they deem appropriate. }\end{array}\end{array}$


consented using a verbal consent form distributed through email and/or in hard copy form. The verbal consent form outlines the purpose of the study and that participation is voluntary. Investigators trained in subject recruitment will ensure the potential participants read and understand the form, and agree to participation before engaging subjects in research. A waiver for the documentation of signed consent was obtained as a further level of protecting VHA staff participants' anonymity. Patients will be consented through a signed consent process and asked to sign a Health Insurance Portability Accountability Act form (a form required by the US law to protect personal health information and medical records) to allow researchers to access their electronic health record. If at any point a potential or consented participant expresses a desire to not participate, investigators will discontinue recruitment or data collection efforts with them.

\section{Data collection}

We will gather and organise preliminary data before the site visit to delimit the organisational context and identify particularly promising areas for interviews and observations. We will visit each facility for a 5-day on-site visit. We will do follow-up data collection, when necessary by phone and protected correspondence. We will undertake to complete roughly one site visit per quarter with 2-2.5 months of qualitative data analysis between. Due to the planning for the ABM (see below), we anticipate that parameters and agent characteristics that we learn about in early interviews will suggest questions and observations for subsequent site visits, checking for the presence or absence of these parameters or agent characteristics. Specific time frames and methods used will be responsive to local context and what we learn during previous site visits.

Team investigators hold advanced degrees in a diversity of fields, including medicine (JP, LL), anthropology (EF, LP), psychology (PN) and business (HL, LL). They each have at least 10 years of experience conducting qualitative research. If not already experienced with complexity theory and $\mathrm{ABM}$, each was provided orientation to these approaches before the study commenced.

\section{Case data collection}

Each site visit will follow the same general data collection approach, with site-specific variations depending on local context (eg, care transition processes, staffing and roles) (table 3). Preparation will involve logistical activities and data gathering through leadership interviews and chart reviews. The 5-day site visit will include a continuation of activities started before the site visits, as well as additional interviews, observations of care transition work, focus groups and staff surveys. Follow-up patient interviews will occur about a month after the site visit.

Throughout the course of case study data collection, team members will talk about what they are finding and fine-tune questions and approaches so that data collection is responsive to site processes and contexts. Decision-making during weekly meetings will be documented in detailed meeting notes. Changes in data collection will be recorded in site-specific data protocol.

Each site visit will be made by three investigators trained and experienced in qualitative methods (JP, PN, LP and/ or HL). Investigators have no relationship with participants prior to the start of the study. Data collection instruments will be tested at the investigators' home facility to ensure inter-rater reliability.

For each case study, qualitative and quantitative data will be collected in the form of background documents, patient chart reviews, semi-structured interviews, focus groups, observations, checklists, debriefments and surveys (table 4).

\section{Qualitative data analysis}

For each case study, qualitative analysis will overlap with data collection processes. Early findings will inform site-specific adjustments to on-site data collection protocols. Qualitative data analysis will take two forms: memoing and coding.

\section{Memoing}

The team will keep a variety of memos during data collection and analysis (table 5). Memos record reflexive comments about methods, data and theory. ${ }^{31}$ Memos will provide early opportunities for writing about and making connections within the case study data. Some memos will be written by individual researchers (eg, chart

\begin{tabular}{|c|c|c|c|}
\hline \multicolumn{4}{|c|}{ 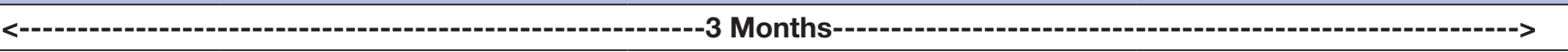 } \\
\hline & Presite visit & 5-Day site visit & Postsite visit \\
\hline Data collection & $\begin{array}{l}\text { Facility background } \\
\text { Chart reviews } \\
\text { Leadership interviews }\end{array}$ & $\begin{array}{l}\text { Leadership interviews (cont.) } \\
\text { Frontline provider interviews } \\
\text { Patient interviews } \\
\text { Focus groups } \\
\text { Observations } \\
\text { Frontline provider surveys } \\
\text { Care transition process checklist }\end{array}$ & $\begin{array}{l}30 \text {-Day postdischarge Interviews } \\
\text { with patients }\end{array}$ \\
\hline Data analysis & Chart review memos & $\begin{array}{l}\text { Observation scoring } \\
\text { Team debrief memos }\end{array}$ & $\begin{array}{l}\text { Facility reflection } \\
\text { Qualitative analysis in NVivo } \\
\text { quantitative analysis }\end{array}$ \\
\hline
\end{tabular}




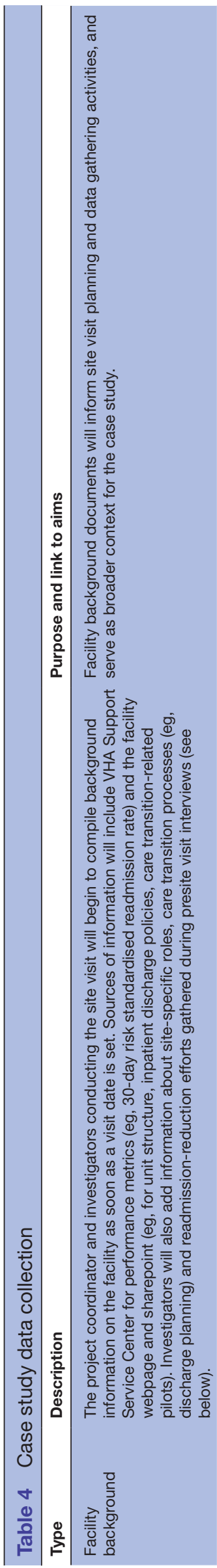

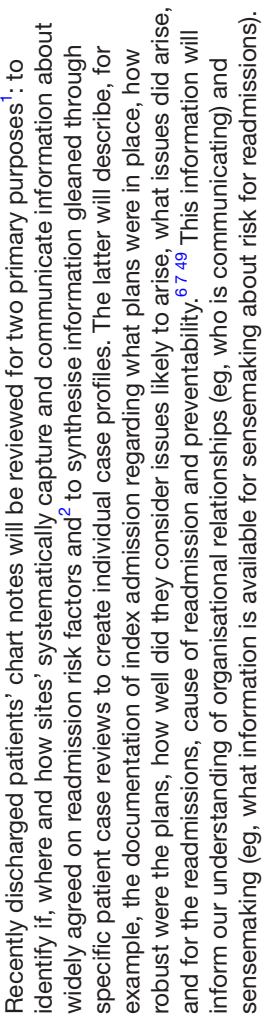

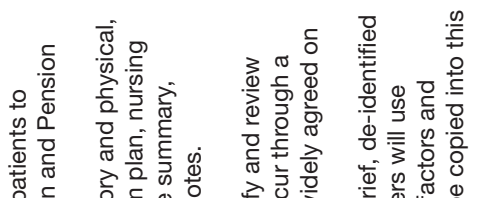

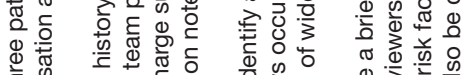

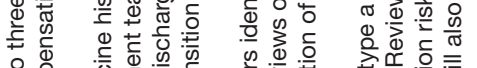

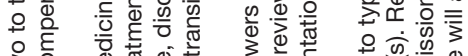

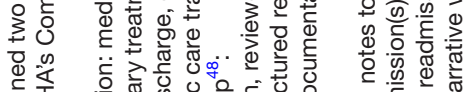

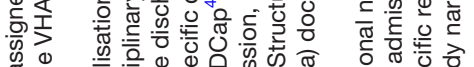
o

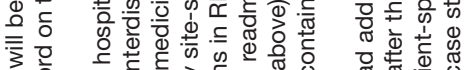
势

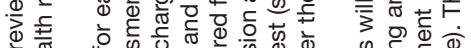

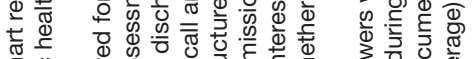

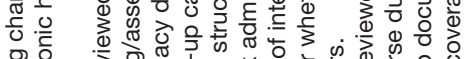
일

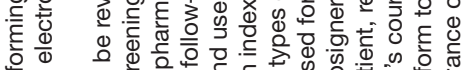

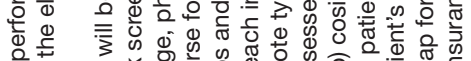

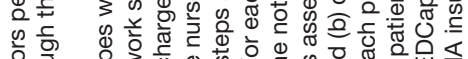

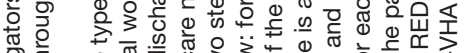
㛃焉 क्ष

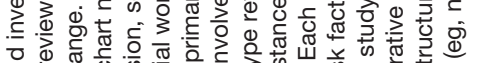

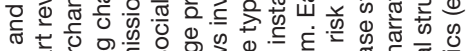

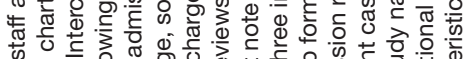

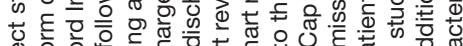

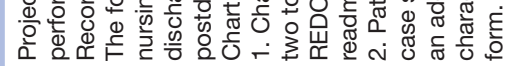

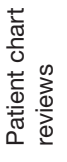

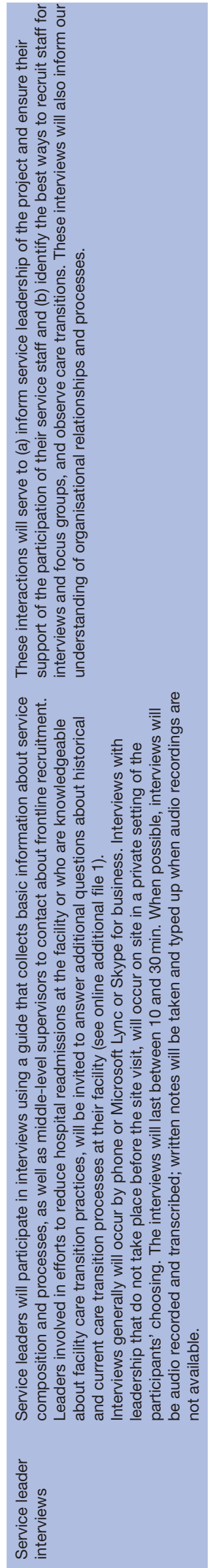

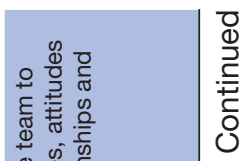

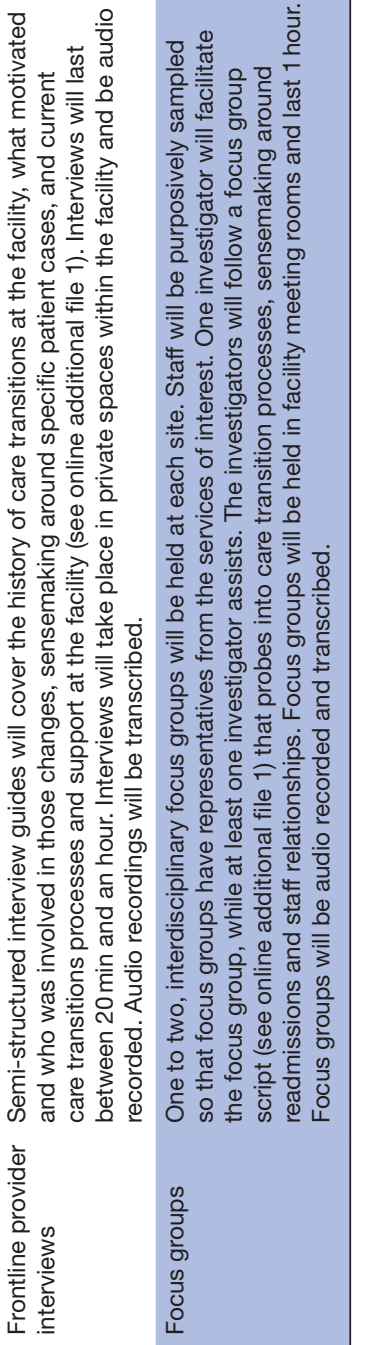




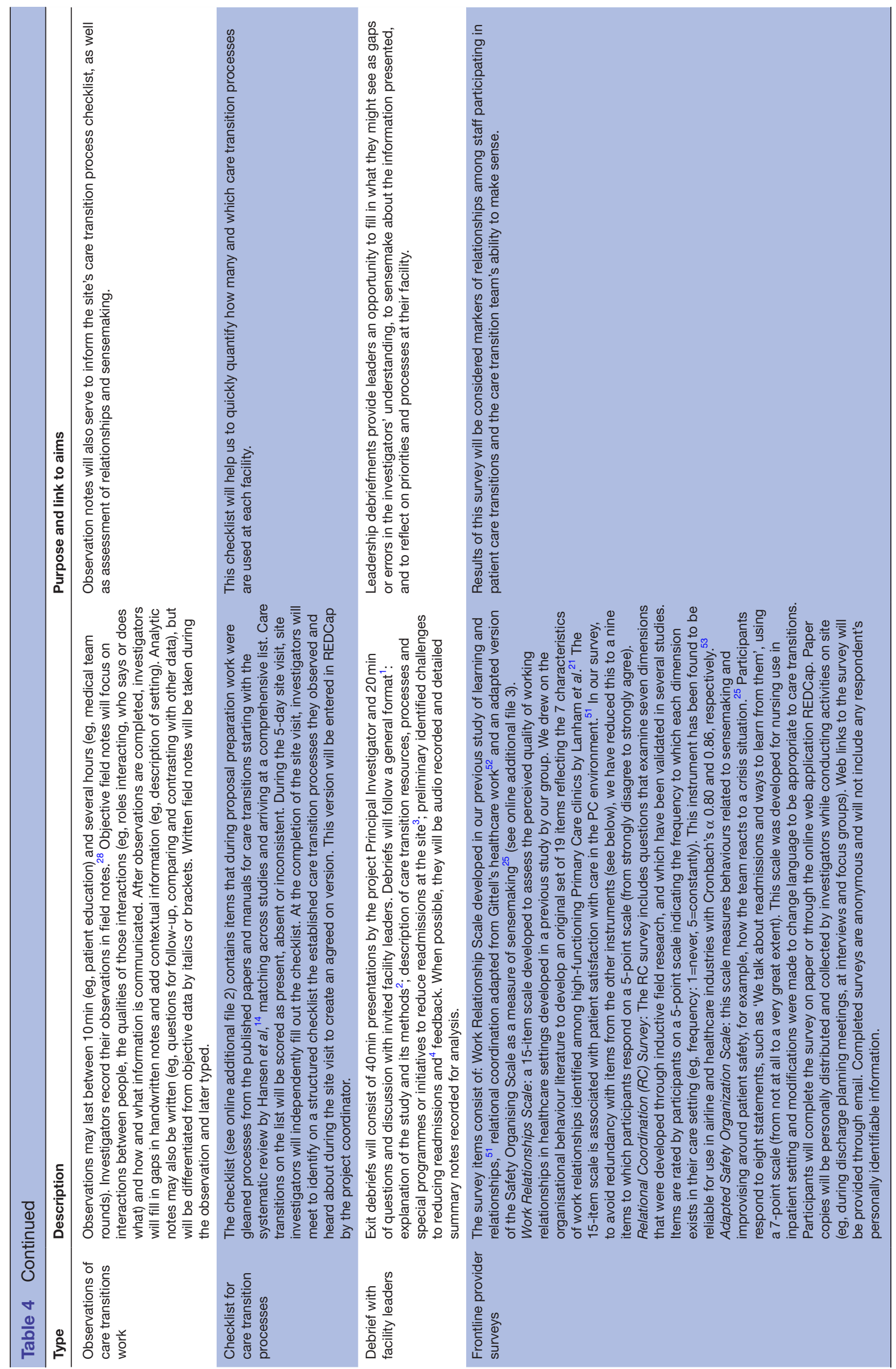


Table 5 Memo types

\section{Memo type Description}

Meeting Detailed summary meeting notes will be kept during team meetings. As described by Eisenhardt, ${ }^{28}$ team meetings memos can be useful for overlapping data collection and analysis. These meeting notes will document, eg, how and why data collection protocols change, what researchers are learning about a specific site and how what they are learning informs theory and agent-based model building. This information will be extracted as memos.

Chart While conducting chart reviews, researchers will write memos to record and reflect on (a) care transition review processes evident in the notes (eg, readmission risk assessment, discharge education, postdischarge follow-up), memos (b) provider communication (eg, cosigning practices, discrepancies in what providers report), (c) sensemaking (eg, providers documented concerns, how patients' situations are described) and (d) questions or issues for team follow-up. These memos will serve to help the team document what they know so far about care transition processes at the site, identify questions for follow-up and reflect on specific cases and provider relationships and sensemaking.

\begin{tabular}{|c|c|}
\hline $\begin{array}{l}\text { Facility } \\
\text { reflections }\end{array}$ & $\begin{array}{l}\text { These one to two page documents will be written by investigators conducting the site visits during postvisit } \\
\text { meetings. Reflections will be organised by headings derived from the agent-based model. These headings will } \\
\text { evolve as the agent-based model develops (see below). Examples of possible headings include: institutional } \\
\text { history and leadership, structures and routines and information flow and exchange. } \\
\text { These analytic memos }{ }^{31} \text { document and summarise what the team thinks they know about the site, what patterns } \\
\text { they observed during data collection and what gaps might exist in their knowledge. Site reflections will inform } \\
\text { the final site case study, data collection methods and approaches at future sites and ongoing analysis and model } \\
\text { building (see below). }\end{array}$ \\
\hline
\end{tabular}

review memos), while others will be created by several researchers through discussion (eg, meeting memos, facility reflections). Memos will be periodically reviewed at team meetings to inform ongoing data collection, qualitative coding and model building. They also serve to help document team sensemaking.

\section{Qualitative coding}

Transcripts will be analysed using NVivo software. ${ }^{32}$ We will develop a code book using deductive and inductive approaches. An initial codebook will be created based on the original model (figure 1). It will be modified as additional elements and patterns are observed through memoing, code report reading and model building.

Coding will proceed in a stepped fashion. For the first two sites, six team members (LP, JP, PN, HL, EF and the project coordinator) will code all interview and focus group transcripts. For each site, a random sample of $20 \%$ of transcripts will be independently coded by two members of the team. Pairs will check for concordance and discrepancies will be discussed by the team, and the codebook updated as needed in bimonthly coding meetings. For the final seven sites, three team members (HL, the project coordinator and a research assistant) will code the remaining transcripts. They will check for concordance on at least $10 \%$ of a random sample of transcripts for each site. Areas of discrepancy will be discussed and resolved by the full research team during weekly team meetings.

\section{Quantitative data analysis}

Quantitative data analysis will be conducted on data collected through patient chart reviews, staff surveys and observations. Knowing readmission rates can change rapidly, at the end of data collection we will also acquire from the VA data warehouse each site's current 5-year readmission rate trend to ensure each site is correctly categorised (as improving or worsening). We will adjust categorisation as necessary. Statistical tests will be conducted in Stata IC V.14. ${ }^{33}$

\section{Chart notes}

At each site, we will determine the likelihood each note type documents the different readmission risk factors and identify which, if any, providers are usually cosigned to the note. We will evaluate findings across and within note types, and across facilities. Findings will also be compared with qualitative data (eg, interview data related to coordination practices and sensemaking related to readmission risk).

\section{Staff surveys}

The survey's three scales will be scored as described in table 6, and the scores compared between sites. As response rates allow, some within site comparisons may also be made. Results will be triangulated with observation, interview and focus group data.

\section{Observation note scoring}

Within their field notes, site investigators will identify the following types of observations for structured scoring: (1) discharge planning meetings; (2) staff-to-staff interactions and (3) staff-to-patient discharge education. Notes from each observation will be entered into scoring logs and scored according to relationship and sensemaking features (table 7). The scoring systems are based on the system by Lanham and colleagues ${ }^{34}$ and situation, task, intent, concern and calibrate frameworks. ${ }^{35}$ Project staff will enter scoring into REDCap.

Two investigators experienced with applying these frameworks to observations in medical settings (LL and $\mathrm{HL}$ ) will train the team on how to recognise behaviours 
Table 6 Scoring frontline provider surveys

\begin{tabular}{|c|c|}
\hline $\begin{array}{l}\text { Survey } \\
\text { instrument }\end{array}$ & Scoring \\
\hline $\begin{array}{l}\text { Work } \\
\text { Relationship } \\
\text { Scale (WRS) }\end{array}$ & $\begin{array}{l}\text { Due to survey burden and partial overlap with other scales (see below), the original } 15 \text {-item WRS was reduced } \\
\text { to } 9 \text { items based on the original Rasch item analyses and areas of overlap with items on the other scales. } \\
\text { Items } 1,2,4,5,8,9,11,14 \text { and } 15 \text { of the original items were retained and references to clinic were changed } \\
\text { to team. }{ }^{51} \text { A new Rasch item analysis and principal components analysis will be conducted to assure that } \\
\text { unidimensionality has been retained. Total scores will be calculated per respondent (possible range 9-45), } \\
\text { averaged across respondents for each facility and facilities will be compared using SAS PROC Mixed. }\end{array}$ \\
\hline $\begin{array}{l}\text { Relational } \\
\text { Coordination } \\
\text { (RC) Survey }\end{array}$ & $\begin{array}{l}\text { RC scores are first calculated for each individual by summing the scores of all roles (eg, care transitions staff, } \\
\text { inpatient attending, outpatient primary care nurse, etc) for each dimension (eg, frequent communication) and } \\
\text { then dividing by the number of responses. The overall RC score for each participant is derived by calculating } \\
\text { the mean of the seven individual scores (range } 1-5) \text {. }^{53} \\
\text { RC scores at the facility level are calculated for each functional group (eg, care transitions manager, hospitalist } \\
\text { primary care nurse or physician) by calculating the mean of each dimension for all members of the functional } \\
\text { group, and then a facility RC mean. The primary analyses will use the facility mean score, and secondary } \\
\text { analyses will examine variation in RC scores among functional groups (care transitions staff, inpatient } \\
\text { attendings, primary care teams). }\end{array}$ \\
\hline $\begin{array}{l}\text { Adapted } \\
\text { Safety } \\
\text { Organization } \\
\text { Scale }\end{array}$ & $\begin{array}{l}\text { Originally described by Vogus and Sutcliffe }{ }^{54} \text { as a measure of self-reported behaviours enabling a safety } \\
\text { culture in-hospital nursing units. Original respondents were nurses only. Questions } 1,3 \text { and } 4 \text { were used } \\
\text { unmodified. Questions } 2,4,7,8 \text { and } 9 \text { were modified to be focused on care transitions and preventing } \\
\text { readmissions. For example, the original question } 2 \text { was 'we talk about mistakes and ways to learn from them'. } \\
\text { The modified version is 'we talk about readmissions and ways to learn from them'. The original question } 5 \text { was } \\
\text { dropped as it dealt only with inpatient nursing shift report giving. The responses were kept the same. As for the } \\
\text { Work Relationship Scale above, a Rasch item analysis and principal components analysis will be conducted to } \\
\text { assure that unidimensionality has been retained. Total scores will be calculated per respondent (possible range } \\
\text { 8-56), averaged across respondents for each facility and facilities will be compared using SAS PROC Mixed. }\end{array}$ \\
\hline
\end{tabular}

that match these characteristics. Consistency in scoring will be established through use of the codebook and during multiple rounds of team scoring. For the first two sites, during weekly meetings following data collection, a sample of roughly $5 \%$ of the observations will be independently scored by each team member. Scoring will be compared and discrepancies discussed until the group has reached consensus. Clarifying discussions about scoring will be documented in meeting notes and fed back to improve the scoring guide. Visual inspection of the distribution of all variables will be performed. Where appropriate, power transformations will be applied to variables outside of assumptions of parametric statistics. Group differences will be determined using ordinary or generalised least squares regression with the relevant covariates.

\section{Objective 2. Creating, verifying and validating an ABM of sensemaking regarding transitions of care and prevention of readmissions}

Complex, non-linear systems are difficult to study with traditional analytic methods because of multiple interactions among variables, feedback loops, path dependency and contingencies in any dynamic process; there is often no set of equations that can be solved to predict characteristics of the system. ${ }^{36}$ A more effective way to examine non-linear behaviour in complex systems is to simulate it by building a model and then running the simulation multiple times to explore the space of possible system trajectories. ${ }^{36}$ In our study of sensemaking and readmissions, the interdependencies among the patients, healthcare providers, resources (VHA and non-VHA) and leadership support are clearly non-linear. Individuals who make sense of the ways in which readmissions occur illustrate this by mentioning different aspects they consider to be critical: patient context, patient understanding and motivation, resource availability, effective communication between healthcare providers, stage of disease, failures in a system for which they (patient or provider) have little control. These aspects interact in variable ways in the context of different patients. Vest et al identified the plethora of variables that contribute to readmissions before even addressing the interdependencies. ${ }^{37}$ Additionally, the literature demonstrates that classical prediction models of readmissions perform poorly. ${ }^{12}$ We suggest that these explanatory gaps in the literature are due at least in part to a mismatch of analytic strategy to type of system being studied. We see readmission as an emergent outcome of non-linear interactions among these many aspects of clinical and organisational processes. Through modelling and simulation, we will be better able to understand and evaluate factors contributing to readmissions. While any single case may be difficult to predict, modelling will allow us to identify leverage points in the system that the data demonstrate are particularly sensitive to sensemaking effectiveness. These leverage points could then be considered potential targets for interventions. Through modelling and 
Table 7 Relationship and sensemaking characteristics to be scored during observations

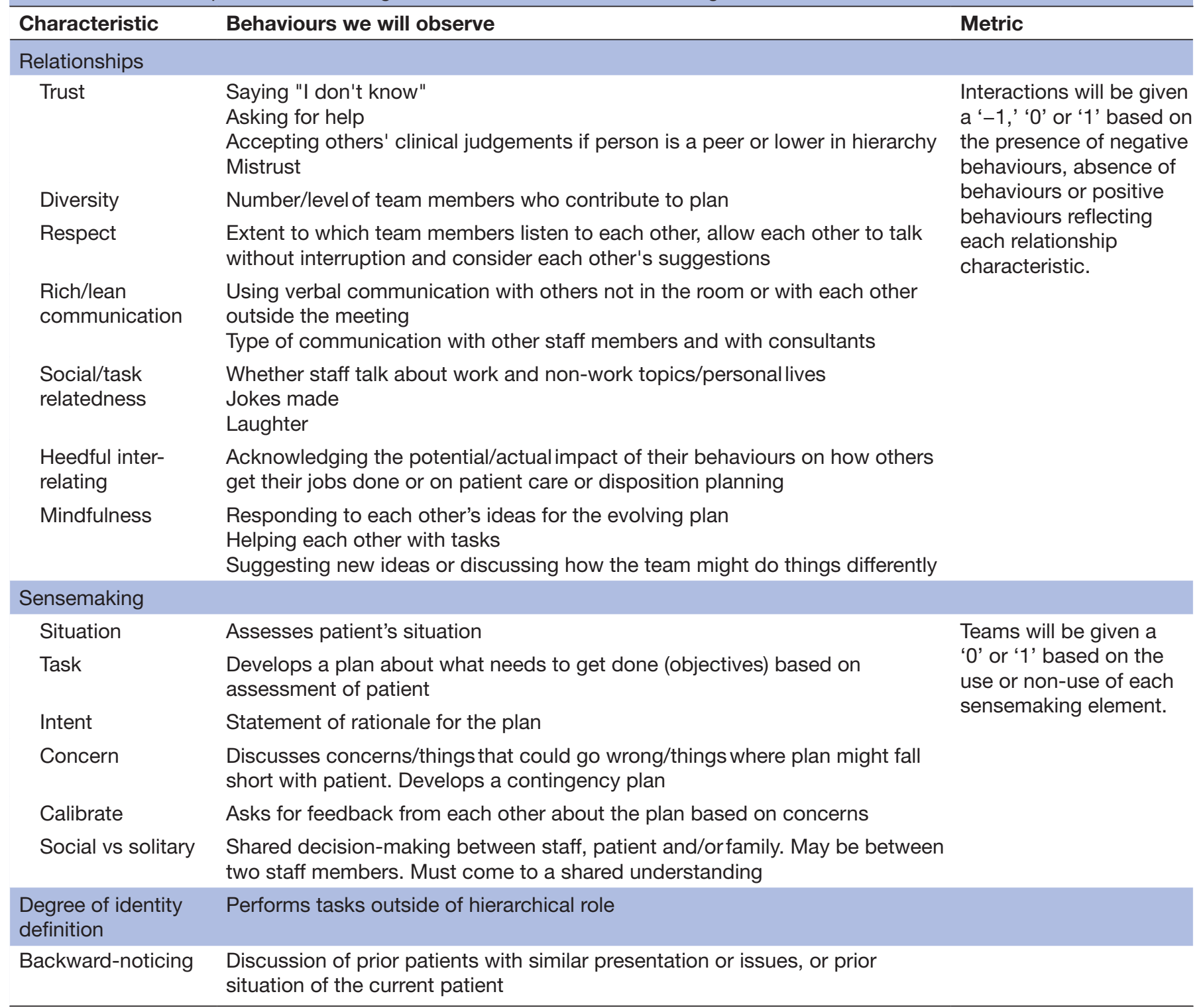

the subsequent ability to run it numerous times (simulation), we will be able to extend the case study sample to make it more generalisable to better understand how readmissions occur across the care transition interventions, patient circumstances and facility environments. Through modelling and simulations we are able to create a laboratory that will allow us to understand better how readmissions occur, helping us to identify gaps in our knowledge as well.

ABM is a version of non-linear dynamic modelling, a computer implementation of complexity concepts, in which autonomous agents interact in an environment to produce emergent-sometimes surprising-system properties over time. ${ }^{38-40}$ Since the pioneering work by Epstein and Axtell in the late 1990s, ${ }^{41}$ it has been applied to research on human groups under the rubric of 'artificial societies'. ${ }^{36} \mathrm{ABM}$ is an ideal approach to our research questions for several reasons: first, as noted earlier, our data regarding healthcare provider interactions are non-linear, making it potentially more difficult to represent patterns and interdependencies using more traditional approaches. ABMs are grounded in non-linear mathematics, assuming interactions and contingencies in a manner that more accurately reflects clinical systems. Second, ABMs allow us to create a broader space of outcomes from rich observations that may be low in number but high in information, accounting for the facilities and teams within facilities that we sample, and other types of findings that result from experimenting with parameter changes. Formalising the interactions leads to a generalisation of the processes we observed. Thus, ABMs enable us to leverage small samples to create broader understandings. Third, we can model interactions across levels and over time to explore emergent outcomes. ABMs are laboratories for structure-agency interactions that allow us to understand these multiple levels. 


\section{Proposed modelling work}

Conceptual work

While data are being collected, our research team will meet regularly to identify the parameters, agent characteristics and interaction patterns. Our starting point will be the conceptual model of care transitions shown in figure 1 . As we develop the ABM, we will iteratively build on our conceptual model using the qualitative data being collected. We will begin developing the ABM after our first few site visits, and refine the model with each subsequent visit. Constructing the model in this way will complement our qualitative data collection and help us identify areas where more intensive inquiry might be necessary. Initial tasks for building the model will include identification of the following:

\section{Types of agents to be included}

In $\mathrm{ABM}$ agents can and, in our case, will have correspondence to real-world actors, both individuals and organisational units. We will start with the general categories of patients, inpatient providers, outpatient providers and care transitions personnel. We will then refine the specific individuals contained in these categories, and add any additional categories or types of individuals as we collect and analyse our qualitative data.

\section{Interactions and interdependencies among agents}

We will create rules of interaction between the agents in the model based on our site visit data, starting with the initial site visits and refining these interactions with subsequent site visit data. Interactions will focus on the sensemaking activities and categories we observe in the site visits. These sensemaking attributes are detailed in the'Observations of care transitions work' and 'Qualitative data analysis' sections.

\section{Boundaries and characteristics of the environment}

Our model will be built to simulate a single organisational entity. We will create a model to allow ourselves the ability to adjust these characteristics and assess their impact through our simulations. We intend to simulate critical facility characteristics and will use the first year to consider the types of qualitative characteristics we will obtain during the site visits as well as the quantitative data already available for VHA facilities such as culture (annual employee survey), learning and improvement culture (voice of VHA survey), number of care transition processes used routinely (from our prior UM survey and verification for study sites), demographics of veterans served and facility admission rates. We will also consider known parameters used in traditional readmission prediction models, although most of these parameters focus on the patient such as comorbidities, prior healthcare use, functional status, socioeconomic status. ${ }^{12} 37$ Organisational characteristics relate back to the technical processes of care and system resources noted on our conceptual model.
Levels of model

One of the rationales in studying transitions of care as an exemplar is the multiple individuals and teams that interact with the patient and the system to make the care transitions successful. A benefit of ABM is that it allows us to consider levels of interactions, and the system-level outcomes that emerge from these levels of interactions. In building the model, we will need to address how different parts interact with the next to produce the product of interest-successful or unsuccessful care transitions. Care transition teams and Veterans interact with inpatient teams as well as outpatient teams, resource providers (such as prosthetics and pharmacy), home care providers, institutional providers and patient caregivers. Additionally, leadership determines extent of resources available at many of these levels. We will define the levels and how they will feed into each other. Again, we will use our conceptual model of care transitions as the starting point. Processes of care and the organisational characteristics will form this level. The formal interactions or organisational structure will also be reflected here. The agents will interact in this level, producing emergent outcomes of sensemaking that are grounded in their interactions and inter-relating. These sensemaking patterns will form the second level of the model. From them, care transition outcomes will emerge, forming the model outputs. In our model, the two outcomes will be a successful care transition or a readmission.

Feedback loops can be created within the levels of the model. For example, as either successful care transitions or readmissions occur, these outcomes can feed back into how the agents' sensemaking processes. We will specifically collect data on these types of feedback loops during our site visits. (See table 4 and additional file 1.) These feedback effects will be modelled using standard best practices from the system dynamics modelling methodology, which concentrates on how to model systems with non-linear feedback loops. ${ }^{42-44}$

\section{Modelling software:}

We will use NetLogo software to create our model. NetLogo is a freely available software that has been under development for two decades and is widely used for ABM. ${ }^{45}$ It is now in V.5 and has become a sophisticated language for modelling intelligent autonomous agents interacting in 'live' environments. With the most recent versions, NetLogo extensions have been incorporated that enable more sophisticated agents and with hybrid capabilities enabling combined agent-based and discreteevent simulation. These capabilities will allow us to create a robust model that best represents the relevant processes of care and agent interactions.

\section{Model verification and refinement}

As we develop the model, we will make our understanding of the interdependencies between different levels more explicit. Because we will begin to conceptualise and create the model in parallel with data collection, we will be able to use ongoing site visits to refine aspects of our model. 
Additionally, we will perform verification to ensure that the associations and interdependencies between levels of the model are expressed in the way we intend. Verification 'concerns whether the programme is working as the researcher expects it to' ${ }^{36}$ Our model will act as a thought-experiment laboratory that forces us to clarify and formalise the interactions in which we are interested. The verification will support this clarification.

\section{Model simulation and sensitivity testing}

We will use simulation to deepen our understanding of the ways that provider sensemaking influences care transition outcomes. We will be able to vary the following parameters: organisational factors, including patient population characteristics and other facility-level data; care transition practices; sensemaking practices. We will assess the impact of parameter variation on our outcome of interest-readmissions and successful care transitions. During this time, simulations will be run for multiple 'facilities' to expand the generalisability of our qualitative sample, using different combinations of individual and facility characteristics to understand how sensemaking emerges, and how sensemaking then impacts care transition outcomes.

\section{Model verification and boundary testing}

During this period, we will present our model results to our local site PIs from 10 sites as well as our systems re-engineering organisational partners for input as to the face validity of the findings of the simulations. These presentations will follow a formal, focus group process to ensure that we capture all concerns and feedback regarding the model. We will use this feedback to further refine the model.

\section{Study status}

Data collection at the first case study site began in July 2015 and continued through December 2017. Qualitative and quantitative data analysis, and ABM work began during this period and were ongoing at the time of writing.

\section{Ethics and dissemination}

Participation in this study is voluntary and participants are not compensated for their participation. Written consent and Health Insurance Portability and Accountability Act forms are obtained for patients participating in interviews. As permitted by our Institutional Review Board, VA staff participating in research activities (eg, interviews, surveys, observations) are given an information form about the study, assured confidentiality and asked to give verbal consent to participation.

Findings from our work will be disseminated through manuscripts in peer-reviewed journals, at professional conferences and in short reports distributed to stakeholders and study participants. Our data will not be made available in repositories.

Contributors JP, LKL, HJL, PN and EPF provided conceptual and methodological expertise to the design of the study protocol. JP and LSP were major contributors to writing the manuscript. All authors read, edited and approved the final manuscript.
Funding This work was supported by Investigator Initiated Research (IIR) Award \#13-040 from the US Department of Veterans Affairs Health Services Research and Development Service.

Competing interests None declared.

Patient consent Not required.

Ethics approval Institutional Review Board (IRB) of the University of Texas Health Science Center at San Antonio.

Provenance and peer review Not commissioned; externally peer reviewed.

Open Access This is an Open Access article distributed in accordance with the Creative Commons Attribution Non Commercial (CC BY-NC 4.0) license, which permits others to distribute, remix, adapt, build upon this work non-commercially, and license their derivative works on different terms, provided the original work is properly cited and the use is non-commercial. See: http://creativecommons.org/ licenses/by-nc/4.0/

C Article author(s) (or their employer(s) unless otherwise stated in the text of the article) 2018. All rights reserved. No commercial use is permitted unless otherwise expressly granted.

\section{REFERENCES}

1. Plsek PE, Greenhalgh T. The challenge of complexity in health care. BMJ 2001;323:625.

2. Bar-Yam Y, Ramalingam C, Burlingame L, et al. Making things work: solving complex problems in a complex world. Cambridge, MA: NECSI, Knowledge Press, 2004.

3. Nuti SV, Qin L, Rumsfeld JS, et al. Association of admission to veterans affairs hospitals vs non-veterans affairs hospitals with mortality and readmission rates among older men hospitalized with acute myocardial infarction, heart failure, or pneumonia. JAMA 2016;315:582.

4. Kocher RP, Adashi EY. Hospital readmissions and the Affordable Care Act: paying for coordinated quality care. JAMA 2011;306:17945 http://jama.jamanetwork.com/article.aspx?doi=.

5. Ashton CM, Wray NP. A conceptual framework for the study of early readmission as an indicator of quality of care. Soc Sci Med 1996;43:1533-41.

6. van Walraven $C$, Bennett $C$, Jennings $A$, et al. Proportion of hospital readmissions deemed avoidable: a systematic review. CMAJ 2011;183:E391-E402.

7. van Walraven C, Jennings A, Forster AJ. A meta-analysis of hospital 30-day avoidable readmission rates: 30 -day avoidable readmission rates meta-analysis. J Eval Clin Pract 2012;18:1211-8.

8. Auerbach AD, Kripalani S, Vasilevskis EE, et al. Preventability and causes of readmissions in a national cohort of general medicine patients. JAMA Intern Med 2016 http://archinte.jamanetwork.com/ article.aspx?doi=10.1001/jamainternmed.2015.7863 (cited 18 Mar 2016).

9. Zuckerman RB, Sheingold SH, Orav EJ, et al. Readmissions, observation, and the hospital readmissions reduction program. $N$ Engl J Med 2016;374:1543-51.

10. Kaboli PJ, Go JT, Hockenberry J, et al. Associations between reduced hospital length of stay and 30-day readmission rate and mortality: 14-year experience in 129 Veterans Affairs hospitals. Ann Intern Med 2012;157:837.

11. Pugh JA, Wang CP, Espinoza SE, et al. Influence of frailty-related diagnoses, high-risk prescribing in elderly adults, and primary care use on readmissions in fewer than 30 days for veterans aged 65 and older. J Am Geriatr Soc 2014;62:291-8.

12. Kansagara $D$, Englander $H$, Salanitro $A$, et al. Risk prediction models for hospital readmission: a systematic review. JAMA 2011;306:1688.

13. Zhou H, Della PR, Roberts $P$, et al. Utility of models to predict 28-day or 30-day unplanned hospital readmissions: an updated systematic review. BMJ Open 2016;6:e011060.

14. Hansen LO, Young RS, Hinami K, et al. Interventions to reduce 30-day rehospitalization: a systematic review. Ann Intern Med 2011;155:520.

15. Leppin AL, Gionfriddo MR, Kessler M, et al. Preventing 30-day hospital readmissions: a systematic review and meta-analysis of randomized trials. JAMA Intern Med 2014;174:1095.

16. Sahay A, Heidenreich PA. A Blended Facilitation To Implement the VA Hospital-to-Home (H2H) Initiative: CHF QUERI. J Card Fail 2011;17:S77.

17. Cilliers P. Complexity and Postmodernism: Understanding Complex Systems. New York: Routledge, 1998.

18. Han PK, Klein WM, Arora NK. Varieties of uncertainty in health care: a conceptual taxonomy. Med Decis Making 2011;31:828-38. 
19. Leykum LK, Parchman M, Pugh J, et al. The importance of organizational characteristics for improving outcomes in patients with chronic disease: a systematic review of congestive heart failure. Implement Sci 2010;5:66.

20. Zimmerman B, Lindberg C, Plsek PE. Edgeware: insights from complexity science for health care leaders. United States of America: York University, 1998.

21. Lanham HJ, McDaniel RR, Crabtree BF, et al. How improving practice relationships among clinicians and nonclinicians can improve quality in primary care. Jt Comm J Qual Patient Saf 2009;35:457-AP2.

22. Miller WL, Crabtree BF, Nutting PA, et al. Primary care practice development: a relationship-centered approach. Ann Fam Med 2010;8 Suppl 1:S68-S79.

23. Dixon-Woods M, Bosk CL, Aveling EL, et al. Explaining michigan: developing an ex post theory of a quality improvement program: an ex post theory of a quality improvement program. Milbank $Q$ 2011;89:167-205.

24. Weick KE. Sensemaking in organizations. 3: Sage, 1995.

25. Blatt $R$, Christianson MK, Sutcliffe $K M$, et al. A sensemaking lens on reliability. J Organ Behav 2006;27:897-917.

26. Haidet P. Jazz and the 'art' of medicine: improvisation in the medical encounter. Ann Fam Med 2007;5:164-9.

27. McKenna K, Leykum LK, McDaniel RR. The role of improvising in patient care. Health Care Manage Rev 2013;38:1-8.

28. Eisenhardt KM. Building theories from case study research. Acad Manage Rev 1989;14:532-50.

29. Pettigrew AM. Longitudinal field research on change: theory and practice. Organization Science 1990;1:267-92.

30. Palinkas LA, Horwitz SM, Green CA, et al. Purposeful sampling for qualitative data collection and analysis in mixed method implementation research. Adm Policy Ment Health 2015;42:533-44.

31. Miles MB, Huberman AM, Saldaña J. Qualitative data analysis: a methods sourcebook. Thousand Oaks, Califorinia: SAGE Publications, Inc, 2014.

32. QSR International. NVivo qualitative data analysis software: QSR International Pty Ltd, 2016.

33. StataCorp. Stata statistical software: release 14. College Station, TX: StataCorp LP, 2015.

34. McAllister $\mathrm{C}$, Leykum LK, Lanham $\mathrm{H}$, et al. Relationships within inpatient physician housestaff teams and their association with hospitalized patient outcomes. J Hosp Med 2014;9:764-71.

35. Leykum LK, Chesser H, Lanham HJ, et al. The association between sensemaking during physician team rounds and hospitalized patients' outcomes. J Gen Intern Med 2015;30:1821-7.

36. Gilbert N, Troitzsch K. Simulation for the social scientist. UK: McGraw-Hill Education, 2005.

37. Vest JR, Gamm LD, Oxford BA, et al. Determinants of preventable readmissions in the United States: a systematic review. Implement Sci 2010;5:88.
38. Railsback S, Grimm V. Agent-based and individual-based modeling: a practical introduction. Princeton University Press: Princeton, 2012.

39. Bonabeau E. Agent-based modeling: methods and techniques for simulating human systems. Proc Natl Acad Sci U S A 2002;99(Suppl 3):7280-7.

40. Macy MW, Willer R. From factors to factors: computational sociology and agent-based modeling. Annu Rev Sociol 2002;28:143-66.

41. Epstein JM, Axtell R. Growing Artificial Societies: Social Science from the Bottom Up: MIT Press, 1996. https://books.google.com/ books?id=xXvelSs2caQC

42. Sterman J. Business dynamics: systems thinking and modeling for a complex world with CD-ROM: McGraw-Hill Education, 2000. https:// books.google.com/books?id=rRmVnQEACAAJ.

43. Sterman JD, Repenning NP, Kofman F. Unanticipated side effects of successful quality programs: exploring a paradox of organizational improvement. Manage Sci 1997;43:503-21.

44. Sastry MA. Problems and paradoxes in a model of punctuated organizational change. Adm Sci Q 1997;42:237.

45. Wilensky U. CCL. NetLogo. 2016 Http://ccl.northwestern.edu/ netlogo.

46. U.S. Department of Veterans Affairs. VHA Support Service Center (VSSC). 2011 vaww.vssc.med.va.gov/cube.asp.

47. Charlson M, Szatrowski TP, Peterson J, et al. Validation of a combined comorbidity index. J Clin Epidemiol 1994;47:1245-51.

48. Harris PA, Taylor R, Thielke R, et al. Research electronic data capture (REDCap)--a metadata-driven methodology and workflow process for providing translational research informatics support. J Biomed Inform 2009;42:377-81.

49. Koekkoek D, Bayley KB, Brown A, et al. Hospitalists assess the causes of early hospital readmissions. J Hosp Med 2011;6:383-8.

50. Kitzinger J. The methodology of Focus Groups: the importance of interaction between research participants. Sociology of Health and Illness 1994;16:103-21.

51. Finley EP, Pugh JA, Lanham HJ, et al. Relationship quality and patient-assessed quality of care in VA primary care clinics: development and validation of the work relationships scale. Ann Fam Med 2013;11:543-9.

52. Gittell JH. High performance healthcare: using the power of relationships to achieve quality, efficiency, and resilience. New York: McGraw Hill, 2009.

53. Gittell JH. Relational coordination: guidelines for theory,

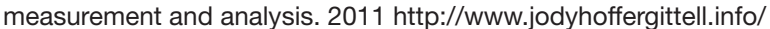
content/rc.html (cited 23 Apr 2011).

54. Vogus TJ, Sutcliffe KM. The safety organizing scale: development and validation of a behavioral measure of safety culture in hospital nursing units. Med Care 2007;45:46-54. 